\title{
Thrombospondin-4 is a putative tumour- suppressor gene in colorectal cancer that exhibits age-related methylation
}

Sonia A Greco', June Chia', Kelly J Inglis ${ }^{1}$, Sarah-Jane Cozzi ${ }^{2}$, Ingunn Ramsnes ${ }^{1}$, Ronald L Buttenshaw ${ }^{1}$, Kevin J Spring ${ }^{1}$, Glen M Boyle ${ }^{3}$, Daniel L Worthley' ${ }^{1}$ Barbara A Leggett ${ }^{1,4}$, Vicki LJ Whitehall ${ }^{1,5^{*}}$

\begin{abstract}
Background: Thrombospondin-4 (THBS4) is a member of the extracellular calcium-binding protein family and is involved in cell adhesion and migration. The aim of this study was to evaluate the potential role of deregulation of THBS4 expression in colorectal carcinogenesis. Of particular interest was the possible silencing of expression by methylation of the $\mathrm{CpG}$ island in the gene promoter.

Methods: Fifty-five sporadic colorectal tumours stratified for the CpG Island Methylator Phenotype (CIMP) were studied. Immunohistochemical staining of THBS4 protein was assessed in normal and tumour specimens. Relative levels of THBS4 transcript expression in matched tumours and normal mucosa were also determined by quantitative RT-PCR. Colony forming ability was examined in 8 cell lines made to overexpress THBS4. Aberrant promoter hypermethylation was investigated as a possible mechanism of gene disruption using MethyLight. Methylation was also assessed in the normal colonic tissue of 99 patients, with samples biopsied from four regions along the length of the colon.

Results: THBS4 expression was significantly lower in tumour tissue than in matched normal tissue. Immunohistochemical examination demonstrated that THBS4 protein was generally absent from normal epithelial cells and tumours, but was occasionally expressed at low levels in the cytoplasm towards the luminal surface in vesicular structures. Forced THBS4 over-expression caused a 50-60\% repression of tumour colony growth in all eight cell lines examined compared to control cell lines. Tumours exhibited significantly higher levels of methylation than matched normal mucosa, and THBS4 methylation correlated with the CpG island methylator phenotype. There was a trend towards decreased gene expression in tumours exhibiting high THBS4 methylation, but the correlation was not significant. THBS4 methylation was detectable in normal mucosal biopsies where it correlated with increasing patient age and negatively with the occurrence of adenomas elsewhere in the colon.

Conclusions: THBS4 shows increased methylation in colorectal cancer, but this is not strongly associated with altered gene expression, either because methylation has not always reached a critical level or because other factors influence THBS4 expression. THBS4 may act as a tumour suppressor gene, demonstrated by its suppression of tumour colony formation in vitro. THBS4 methylation is detectable in normal colonic mucosa and its level may be a biomarker for the occurrence of adenomas and carcinoma.
\end{abstract}

\footnotetext{
* Correspondence: Vicki.Whitehall@qimr.edu.au

'Conjoint Gastroenterology Laboratory, Royal Brisbane and Women's Hospital Research Foundation, Clinical Research Centre and Queensland Institute of Medical Research, Brisbane, Australia

Full list of author information is available at the end of the article
}

\section{() Biomed Central}

(c) 2010 Greco et al; licensee BioMed Central Ltd. This is an Open Access article distributed under the terms of the Creative Commons Attribution License (http://creativecommons.org/licenses/by/2.0), which permits unrestricted use, distribution, and reproduction in any medium, provided the original work is properly cited. 


\section{Background}

Thrombospondin-4 (THBS4) is a member of the extracellular calcium-binding protein family. It is a secreted pentameric globular protein that forms part of the extracellular matrix, and functions in calcium binding, cell attachment, cell migration, cellular proliferation, cytoskeletal organisation, neurite growth, binding of other extracellular matrix components and cell to cell interactions [1-6]. THBS4 is expressed at high levels in the heart and skeletal muscle, is detected in neuronal tissue in the brain and eye, and also in the skin, lung, pancreas, T-cells, breast and colon tissues $[1,3,5,7,8]$. It is a putative tumour suppressor gene, the hypermethylation of which has been linked to cutaneous T-cell lymphoma, breast cancer and colorectal cancer $(\mathrm{CRC})[6,8,9]$.

DNA methylation is the addition of a methyl group to cytosine nucleotide bases via one of the DNA methyltransferase enzymes [10]. CpG islands are most commonly found within the promoter region of genes, although they can occur in other coding and non-coding regions. More than $60 \%$ of gene promoters are found within CpG islands [11]. CpG islands are usually not methylated regardless of the expression of the gene [7]. However, if these promoter $\mathrm{CpG}$ islands become methylated, the associated gene may be permanently silenced, and this silencing is epigenetically inherited $[10,11]$. The promoter region of THBS4 is typical of such a CpG island that is subject to epigenetic silencing. As a putative tumour-suppressor gene, methylation of the THBS4 promoter and silencing of its tumour-suppressor function is potentially pathogenic.

The CpG Island Methylator Phenotype (CIMP) has been used to describe a subset of colorectal tumours with a high frequency of methylation in genes known to be specifically methylated only in neoplasia and not in normal colon [12,13]. While age-related (Type A) promoter methylation of some genes is common in both normal mucosa and neoplastic tissue, there is a subset of genes whose promoters are only found to be hypermethylated in CRC (Type C, cancer-related methylation) [12,14]. However, age-related hypermethylation in normal colonic mucosa may contribute to the pre-malignant colorectal field $[14,15]$.

Sporadic colorectal cancer (CRC) can be divided into clinically relevant subgroups based on gene expression profiles that reflect pathways of tumour progression. The traditional pathway accounts for approximately $70 \%$ of all CRC [13]. These are characterised by chromosomal instability, gene mutations and deletions, and are microsatellite stable (MSS). Adenomas are the precursor lesions to traditional pathway colorectal cancers [16]. The serrated pathway accounts for approximately $30 \%$ of all sporadic CRC. These are characterised by a different spectrum of target gene alterations $[15,17,18]$. Mutations in the BRAF oncogene are common, along with CIMP [19]. Serrated pathway tumours have high levels of microsatellite instability (MSI-H), which results from the hypermethylation and subsequent silencing of the mismatch repair gene $M L H 1$. Serrated polyps precede the development of this tumour subgroup [15].

The aims of this study were to evaluate the deregulation of THBS4 in a series of colorectal cancers, to examine correlations with CIMP, to probe the effect of forcing THBS4 protein expression in CRC cell lines and to examine the mechanism of THBS4 deregulation.

\section{Methods}

\section{Patient Samples}

Quantitative expression (qRTPCR) analysis was performed on 55 matched normal and tumour samples. All tumour samples collected in a fresh state were macroscopically dissected to remove contaminating normal mucosa. Quantitative methylation (qMSP) analysis was also conducted on this patient cohort as well as biopsies of normal mucosa taken during clinically indicated colonoscopy in a series of 99 patients. Biopsies were taken from each of 4 locations within the colon (caecum, transverse colon, sigmoid colon and rectum). All polyps were removed and submitted for histology. All patients gave written, informed consent, and the study was approved by the RBWH and QIMR Human Research Ethics Committees. DNA was extracted from tumour samples using a salt precipitation method as previously described [20], and from biopsy specimens using a DNA column method (Qiagen, Hilden, Germany). CIMP status was determined in tumours by normalising the methylation levels of each of the 5 Laird CIMP markers (CACNA1G, IGF2, NEUROG1, RUNX3, SOCS1) [19] by the methylation levels observed for the highly methylated $A L U$ gene to generate the PMR, or Percentage of Methylated Reference. Tumours were classified as CIMP-negative (CIMP-neg) if 0/5 markers were methylated, CIMP-low (CIMP-L) if $1 / 5$ or $2 / 5$ markers were methylated, and CIMP-high (CIMP-H) if 3,4 or $5 / 5$ markers were methylated. The study cohort consisted of 14. CIMP-High, 11 CIMP-Low and 30 CIMP-Negative tumours.

\section{Expression analysis}

Total RNA was extracted using an RNeasy MidiPrep kit (Qiagen, Hilden, Germany) and cDNA was synthesised using random hexamers and SUPERSCRIPT III (Invitrogen, Carlsbad, California). A Taqman ${ }^{\circ}$ Gene Expression Assay (part\# 4331182; assay ID Hs00170261_m1 Applied Biosystems, Carlsbad CA, USA) was performed on cDNA generating a 96 bp THBS4 product. Gene 
expression was normalised to $\beta$-actin $(A C T B)$ expression using Taqman ${ }^{\circledR}$ Gene Expression Assay (part\# 4331182; assay ID Hs99999903_m1 Applied Biosystems, Carlsbad CA, USA) generating a $171 \mathrm{bp}$ product. The qPCR was performed in duplicate on a RotorGene3000 (Qiagen) using Absolute QPCR Mix (AB1133A; Integrated Sciences, NSW, Australia) and cycling of 40 cycles at $60^{\circ} \mathrm{C}$ annealing. The mathematical model described by Pffafl was used to determine the expression of THBS4 relative to the housekeeping gene $A C T B$ [21].

\section{THBS4 Immunohistochemistry}

THBS4 immunohistochemistry was performed on a subset of 40 patients. Briefly, fixed tumours were embedded in paraffin blocks and $0.2 \mu \mathrm{M}$ sections were cut and mounted onto Superfrost Plus slides. They were deparaffinized in xylol and rehydrated by gradient alcohol before a 15 minute incubation with $0.5 \%$ hydrogen peroxide in phosphate buffered saline (PBS) to quench activity of endogenous peroxidases. After washes in PBS, the first containing $0.05 \%$ Triton X-100, sections were incubated in $10 \%$ goat serum and $0.01 \%$ acetylated BSA for 60 mins, then incubated overnight at $4^{\circ} \mathrm{C}$ in mouse anti-human THBS4 monoclonal antibody (MAB2390, R\&D Systems, Minneapolis, MN) at $1: 1500$ in $\mathrm{PBS} / 5 \%$ goat serum/1\%BSA. Sections were again washed and incubated with rabbit anti-mouse Envision (Dako, Denmark) for 30 mins. The chromogenic substrate was 3,3-diaminobenzidine and sections were counterstained with Meyers' prior to dehydration and mounting of slides in Depex. Negative (no antibody) controls were included in all runs. Once the normal staining pattern for THBS4 was established, positive control normal tissue sections were also included in all runs for consistency.

\section{Colony formation assay}

A mammalian expression construct (pcDNA3.1::THBS4) was designed to examine the effect of over-expressing THBS4 in CRC cell lines. Briefly, $1 \mu \mathrm{g}$ of either pcDNA3.1(+) (Invitrogen, Carlsbad, CA, USA) or pcDNA3.1::THBS4 was transfected into cells using FuGENE6 (Roche Applied Science, Indianapolis, IN) at a ratio of 3:1. Three null-expressing cell lines (Lim1215, SW48 and HT29), one low-expressing cell line (SW480) and four high-expressing cell lines (DLD1, LS174T, HCT116 and RKO) were transfected in triplicate at an initial density of approximately $50 \%$. Cells were allowed to recover for $48 \mathrm{hrs}$ before application of selective media at a final concentration of $700 \mathrm{ng} / \mu \mathrm{L}$ G418 (GibcoBRL Life Technologies, Invitrogen, CA) for 10-14 days. At this time there were no surviving untransfected control cells, and transfected colonies were stained with $0.25 \%$ crystal violet $/ 80 \%$ methanol. The HT29 transfection was repeated and individual colonies were expanded to confirm THBS4 transcript re-expression.

\section{Methylation Analysis}

In order to determine the methylation status of the THBS4 promoter, qMSP was performed for 55 paired normal and tumour samples, and 13 cell lines. Briefly, $1.5 \mu \mathrm{g}$ of DNA was modified with sodium bisulfite using the EpiTect Bisulfite kit (Qiagen, Hilden, Germany), diluted 1/8 and subjected to real time PCR in duplicate on a Rotorgene6000 (Corbett Research, QIAGEN, Germany) using Absolute QPCR Mix (AB1133A; Integrated Sciences, NSW, Australia) and cycling of 40 cycles at $60^{\circ} \mathrm{C}$ annealing (400 nM of F 5'-CGTTGTCGCGGAGTTTAGTA-3'; 600 nM of R 5'-ACGACGACGACGTTAACC-3'; 50 nM of Probe 5'-[DFAM]-ACCTCGATC GACGCCCGAAC [DBHQ1]). The level of methylation was determined by normalising the THBS4 methylation levels by the methylation levels observed for the highly methylated $A L U$ gene to generate the PMR, or Percentage of Methylated Reference (400 nM of ALU-F 5'-GGTTAGGTATAGTGGTTTATATTTGTAATTT-3'; 400 nM of ALU-R 5'ATTAACTAAACTAATCTTAAACTCCTAACCT-3'; $100 \mathrm{nM}$ of ALU-Probe [6FAM]-CCTACCTTAAC CTCCC-[MGBNFQ]; cycling as described for THBS4 qMSP above). Analysis was performed using MethyLight [22].

\section{Statistical Analysis}

The Wilcoxon signed rank test was used to compare THBS4 expression as well as methylation between the normal mucosal and tumour pairs. The Kruskal Wallis test was used to test for a relationship between tumour THBS4 methylation and CIMP status as well as the difference in normal mucosal THBS4 methylation in patients with and without colorectal pathology. A nonparametric trend test was applied to confirm an ordinal gradient across multiple groups. THBS4 methylation within the normal colonoscopic biopsy samples was calculated at each of the four sites sampled, as well as analysed according to an average proximal (mean of cecum and transverse colon), distal (mean of sigmoid colon and rectum) and pancolorectal (mean of all four sites) result. Correlations were analyzed by the Spearman's rank $(\rho)$ coefficient. Distal and proximal levels of methylation were analyzed by a paired t-test. All tests were performed using Stata Statistical Software, version 10 [StataCorp].

\section{Results}

\section{Expression Analysis}

THBS4 expression normalised to $A C T B$ was generally quite low in both peritumoural normal mucosa and in tumours (Figure 1). There was significantly higher 


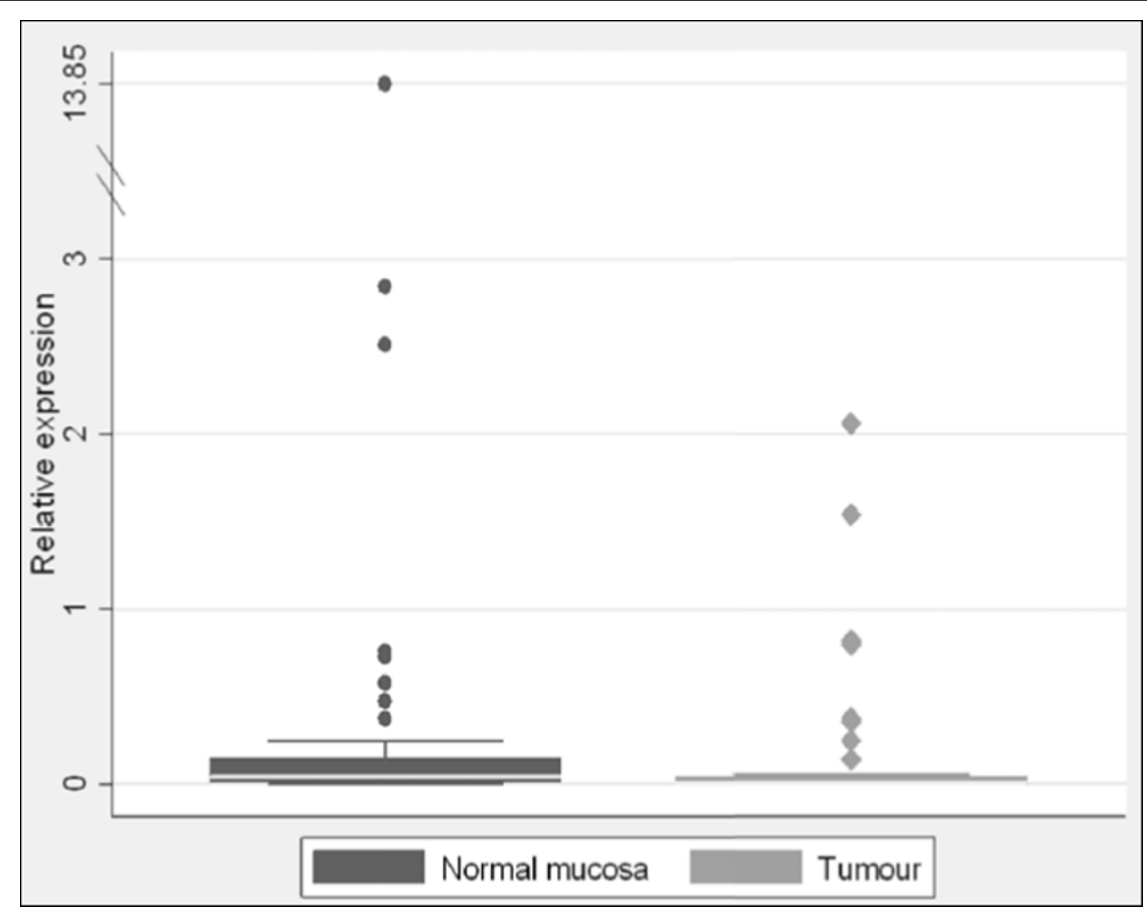

Figure 1 THBS4 expression is higher in normal mucosa than in tumour tissue. While the relative expression of THBS4 was quite low overall, THBS4 expression was significantly higher in peritumoural normal mucosa than in the tumour tissue. Median expression in normal mucosa was 0.04 vs. 0.01 for tumour tissue $(p=0.0035)$. Circular and diamond shaped dots represent individual data point for normal and tumours samples, respectively.

expression of THBS4 in the normal mucosa compared to the tumour. Median THBS4 expression was 0.04 vs. 0.01 respectively $(\mathrm{p}=0.0035)$.

\section{THBS4 Immunohistochemistry}

Figures 2A,B \&2C show the examples of THBS4 immunohistochemical staining. In normal tissue, (Figure 2A and the right side of $2 \mathrm{~B}$ ) THBS4 protein was absent from the majority of normal epithelial cells, but was occasionally expressed in a vesicular pattern. Low levels were seen in the cytoplasm toward the luminal surface. THBS4 expression tended to be even lower in tumours compared with normal mucosa, with the majority of tumours having no detectable THBS4 expression (left side of Figure 2B, Figure $2 \mathrm{C})$. In general, THBS4 protein was most likely to be seen at the luminal surface of normal crypts or tumour glands. However, there was some heterogeneity within the tumour sections and Figures 2D,E and 2F show some interesting variations to the general protein localisation in tumour sections. In Figures 2D and 2E, THBS4 protein seemed to be significantly up-regulated and actively secreted or packaged in vesicular structures (Figure 2F). These expression patterns suggest that THBS4 protein may be secreted or packaged and may give clues as to its function, although further functional studies are now required to test this hypothesis. If THBS4 protein is being packaged and secreted, it is possible that this may be one reason such low levels are detected in normal mucosa. The low frequency of detectable staining in tumour samples did not allow meaningful comparison with other molecular analyses. Immunohistochemical staining was primarily used to provide novel insight into the cellular distribution of THBS4 expression patterns.

\section{Colony formation assay}

Colony formation was significantly repressed in all eight cell lines examined following over-expression of THBS4. We observed that both the number of colonies and their size were greatly reduced upon forced over-expression of THBS4. Figure 3 quantifies the THBS4 repression of colony formation in all cell lines tested. The reduction in colony formation ranged from $34 \%$ to $89 \%$ compared with the vector-only controls. Forced overexpression of THBS4 in multiple colorectal cancer cell lines, regardless of their basal THBS4 expression, consistently reduced colony forming ability. This indicates that high levels of this protein is associated with reduced tumour cell growth. To confirm that THBS4 transcript was introduced by transfection with this construct, HT29 was transfected and 14 individual clones were expanded. Of these, 8 showed high levels of THBS4 transcript whilst the remaining 6 had negligible 

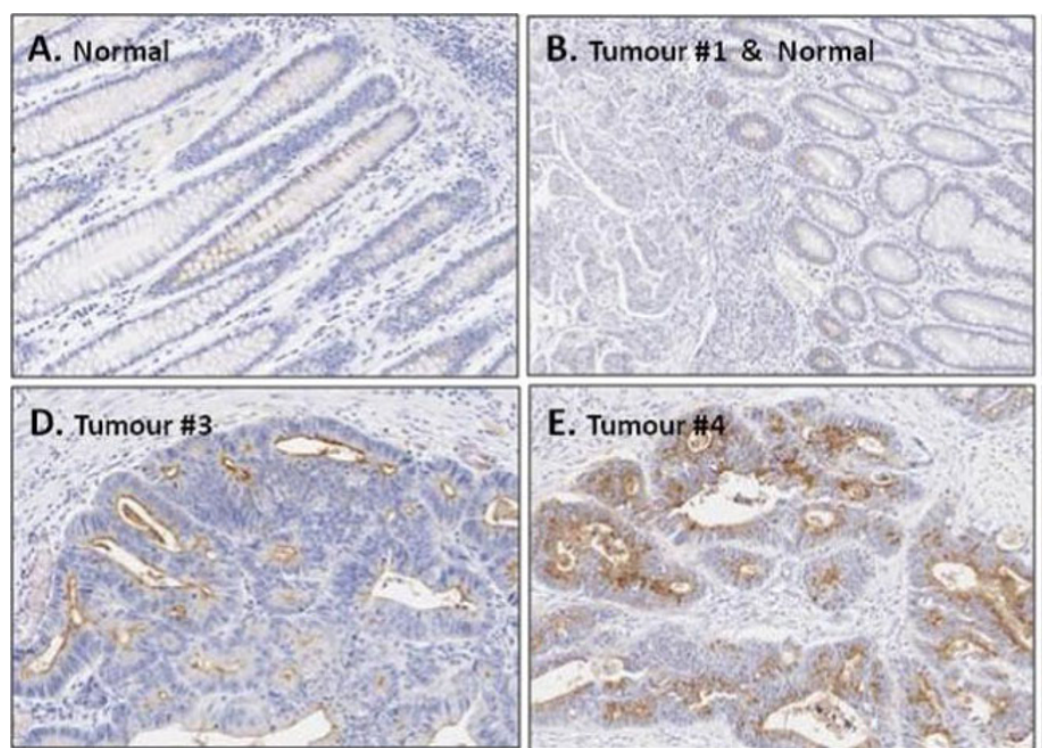
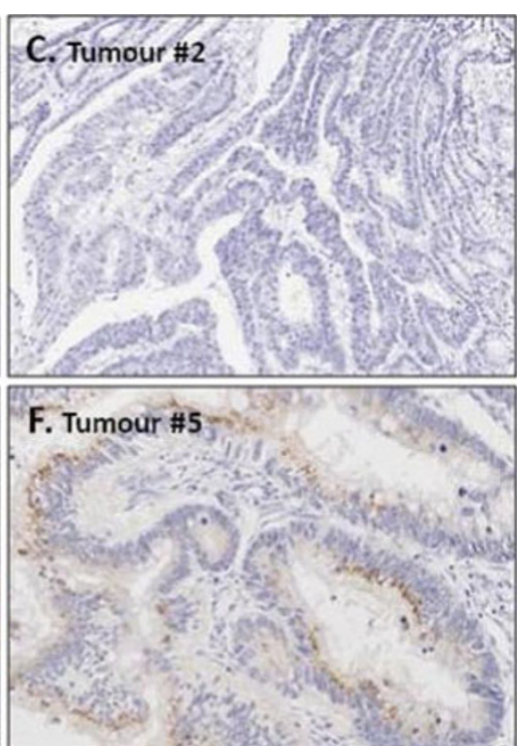

Figure 2 The THBS4 protein appears to be secreted. THBS4 protein is not expressed or is expressed at low levels in normal cells, especially towards the luminal surface. Tumour expression of THBS4 is heterogeneous, with generally no expression but occasional areas of strong expression and packaging in vesicular structures.

expression levels comparable with untransfected parental HT29 cells.

\section{Methylation Analysis}

Overall, colorectal cancers had significantly higher THBS4 methylation than the matched normals (Figure 4). The median PMR in normals was 0.06 vs. 2.6 in tumours
$(\mathrm{P}<0.0001)$. Tumours were classified into CIMP subgroups using the Laird marker panel [19]. When stratified by CIMP status, there was no difference in the methylation of normal mucosa between the different classes of tumours. However, THBS4 methylation in tumours was positively correlated with CIMP (Figure 5). Tumour PMRs showed a progressive increase with increasing level of

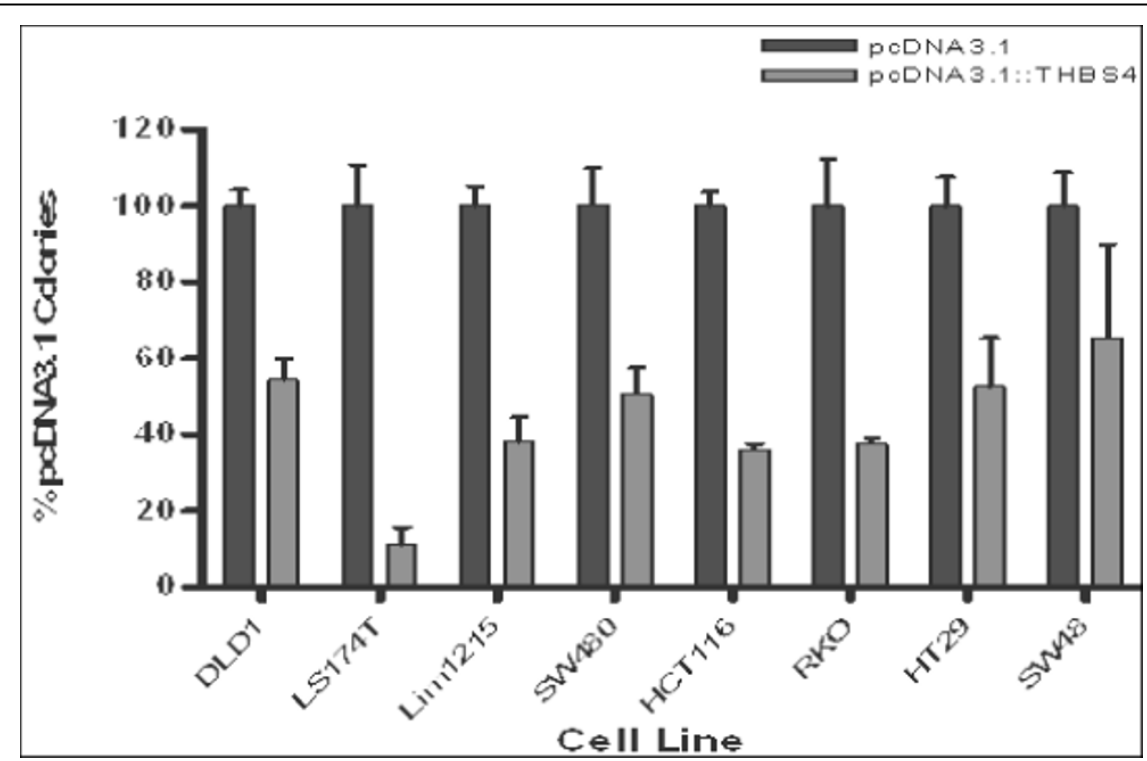

Figure 3 Over-expression of THBS4 significantly decreases colony formation in CRC cell lines. Forced over-expression of THBS4 significantly decreases colony formation in colorectal cancer cell lines regardless of their baseline expression levels. The average reduction in colony formation following THBS4 over-expression was 50-60\% compared with vector-only controls. This consistent and significant reduction in colony forming ability may indicate that high levels of this protein could correlate with reduced tumour cell growth. 


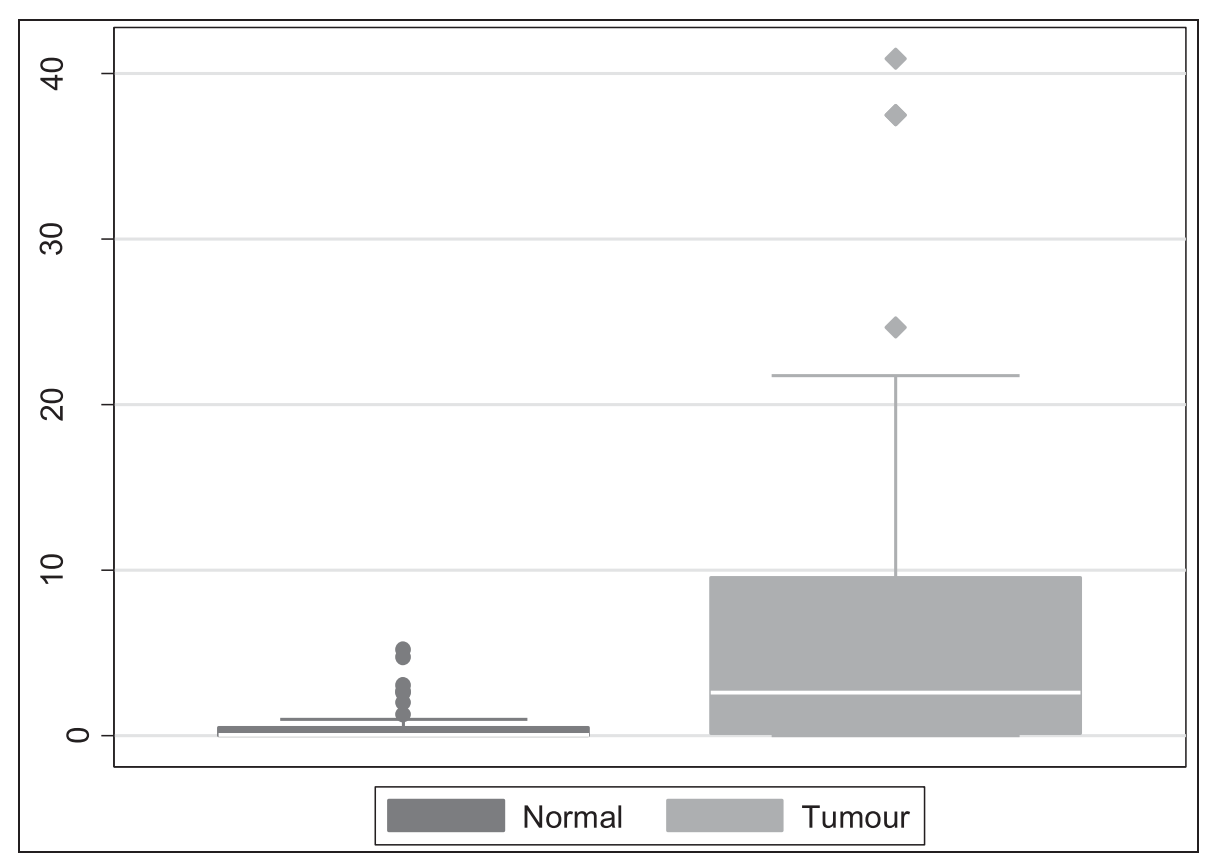

Figure 4 THBS4 methylation is significantly higher in neoplastic tissue compared to adjacent normal tissue. THBS4 methylation was significantly higher in neoplastic tissues compared to the adjacent normal tissue $(p<0.0001)$. The median PMR in normal mucosa was 0.06 compared with a PMR of 2.6 in tumour tissue.

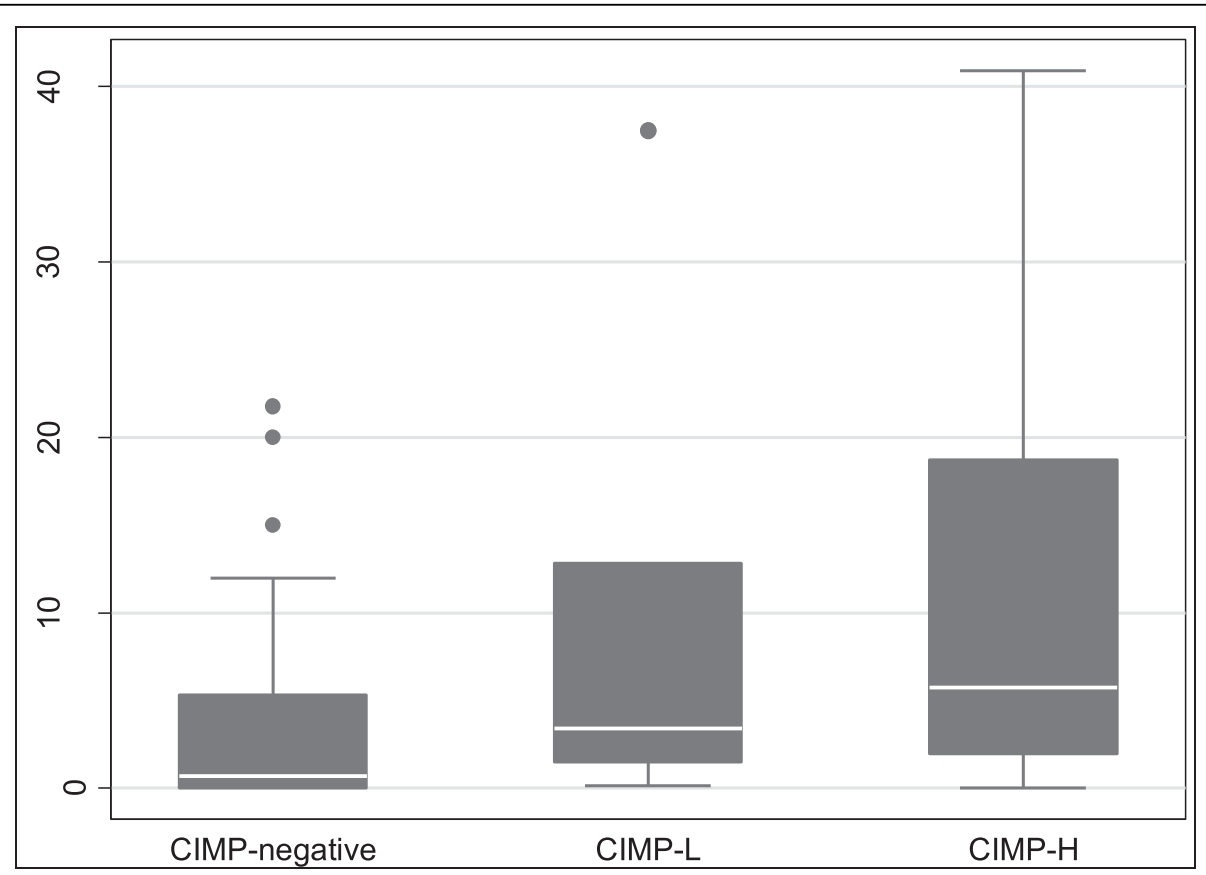

Figure 5 THBS4 methylation increases with increasing CIMP methylation. Tumours were stratified according to the Laird classification of CIMP, separated into CIMP-neg (0/5 markers positive for methylation), CIMP-low (1/5 or 2/5 markers positive for methylation) and CIMP-high ( 3,4 or $5 / 5$ markers methylation positive) categories of methylation. THBS4 methylation was found to positively correlate with CIMP. The greater the number of Laird markers that were positive for CIMP, the higher the level of THBS4 promoter methylation. The median PMRs were 0.69 for CIMP-negative tumours, 3.4 for CIMP-L tumours, and 5.8 for CIMP-H tumours ( $p=0.033$ ). 
CIMP, with the median PMR for CIMP-negative tumours being 0.69, 3.4 for CIMP-L tumours, and 5.8 for CIMP-H tumours $(\mathrm{p}=0.033)$.

Using a PMR of 10 as a cut off [19], tumours were separated into THBS4 methylation positive (PMR $\geq 10$ ) and THBS4 methylation negative (PMR < 10). THBS4 promoter hypermethylation (PMR $\geq 10$ ) was observed in 4/30 (13.3\%) CIMP-NEG tumours, 4/11 (36.4\%) CIMP-L tumours, and 5/14 (35.7\%) CIMP-H tumours. Thus, while the average tumour PMR correlates well with an increasing number of markers positive for CIMP, there were an equal proportion of CIMP-L and CIMP-H tumours that showed high THBS4 methylation.

\section{Correlation of Expression and Methylation of THBS4}

In CRC cell lines, there was no significant correlation between THBS4 transcript expression when compared with THBS4 PMR ( $\rho=0.42, \mathrm{p}=0.20$ ) on a continuous scale (Table 1). There was also no difference in expression in the colorectal cancer cell lines tested when the PMR cut-off of $\geq 10$ was used ( $p=0.35$, data not shown). However, of the 11 cell lines tested, only 3 had a PMR of $<10$. Tumours were also analysed for THBS4 expression level. Tumours with THBS4 PMR $\geq 10$ appeared to have lower THBS4 expression, but this difference was not significant, according to the Wilcoxon Rank-sum (Mann-Whitney) analysis ( $\mathrm{p}=0.26$, data not shown). Thus, while there was a trend towards lower THBS4 expression in tumours with a PMR greater than or equal to 10 having decreased expression, this difference was not significant. When tumour expression levels were normalised to matched normal mucosa expression levels, 44 cancers showed reduced expression greater than 1.5 fold (average 186.5 fold down-regulation) whilst 15 cancers showed greater than 1.5 fold up-regulation

Table 1 THBS4 expression is not related to its promoter methylation in CRC cell lines

\begin{tabular}{lll}
\hline Cell Line & THBS4 Expression & THBS4 PMR \\
\hline DLD1 & 7.24 & 93.68 \\
\hline HCT116 & 7.9 & 124.41 \\
\hline HT29 & 0 & 100.06 \\
\hline LIM1215 & 0 & 0 \\
\hline LIM1863 & 0 & 0 \\
\hline LISP1 & 12.92 & 140.51 \\
\hline LoVo & 0.3 & 14.8 \\
\hline LS174T & 7.24 & 2.23 \\
\hline RKO & 6.97 & 224.29 \\
\hline SW48 & 0 & 139.72 \\
\hline SW480 & 0.89 & 58.6 \\
\hline
\end{tabular}

There is no correlation between reduced THBS4 expression high THBS4 promoter methylation in CRC cell lines ( $\rho=0.42, p=0.20$ ). (average 26.5 fold) and seven tumours did not vary in expression levels by more than 1.5 fold.

\section{Methylation of THBS4 in Normal Colonic Biopsies}

THBS4 methylation was detected within the normal mucosa in all 99 of the colonoscopy patients with similar levels of methylation within the proximal and distal colorectum (median PMR 0.66 vs. 0.65, respectively, $\mathrm{p}=0.66)$. Additionally, there were no differences in THBS4 methylation between the sexes, either at individual sites or in terms of pancolorectal THBS4 methylation. There was however, a strong and direct correlation between patient age and pancolorectal THBS4 methylation $(\rho=0.50, \mathrm{p}<0.0001$, Figure 6$)$.

THBS4 methylation was lower in the background mucosa of patients with colorectal adenomas compared to those with normal colonoscopies. Inclusion of the peritumoural normal mucosal samples and stratification by age, revealed a consistent and inverse association between adenomatous pathology and THBS4 methylation (Figure 7). Importantly, THBS4 methylation within the normal colorectal field showed an impressive and inverse biological gradient with the presence of colorectal pathology, with the lowest levels of THBS4 methylation within the mucosal field being associated with the most advanced pathology (Figure 7). This suggested that THBS4 methylation in the normal mucosa was not directly implicated in promoting colorectal neoplasia and could in fact be directly or indirectly protective. This is supported by our DNA methylation data in other "type A" genes [23].

\section{Discussion}

Thrombospondin-4 is a putative tumour-suppressor gene that plays an integral role in mediating cellular processes such as cell attachment and migration [4]. Classes of tumour suppressor genes include those involved with DNA repair, cell growth, cell cycling, cell adhesion, cell migration, transcriptional regulation and apoptosis [24]. These may be inactivated through gene mutation, chromosomal deletion or methylation [25]. Tumour suppressor genes encode proteins which, upon loss of function, such as through epigenetic silencing, leads to a selective growth advantage for neoplastic cells. THBS4 has shown to be methylated in several tumour types, and this methylation is associated with transcriptional down-regulation $[6,8,9]$.

Thrombospondin-4 is expressed at low levels in normal colonocytes, especially in the cytoplasm towards the luminal surface. Expression in tumours tends to be even lower than the levels observed in normal mucosa. Immunohistochemical localisation of THBS4 confirmed the absence of protein expression in the majority of tumours. However, some cancer cells within particular 


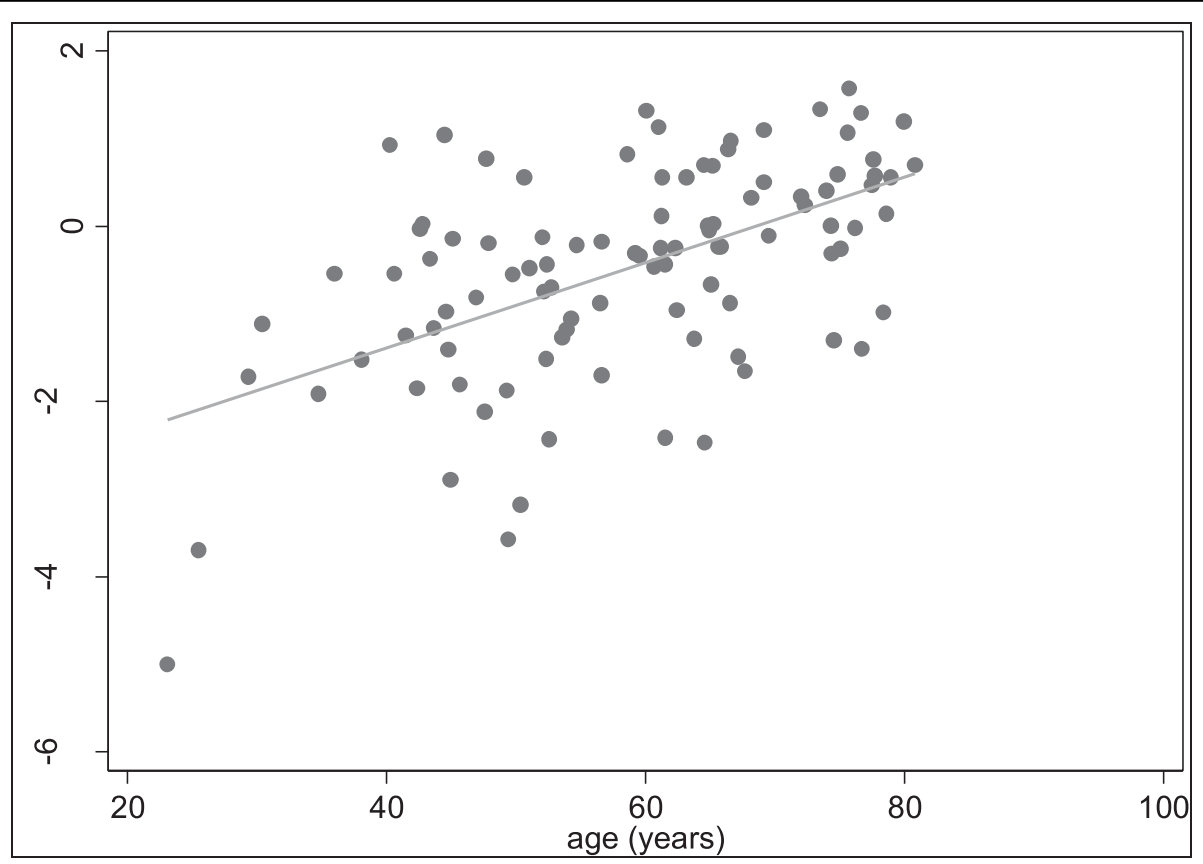

Figure 6 THBS4 methylation is associated with ageing in normal colorectal mucosa. A strong correlation was observed between THBS4 methylation (presented as the log transformed pancolorectal result) and age in the normal mucosa from the colonoscopy study $(\rho=0.50$, $P<0.0001$ ). Because of this strong relationship with aging, we have postulated that THBS4 is a Type A (age-related) marker of methylation.

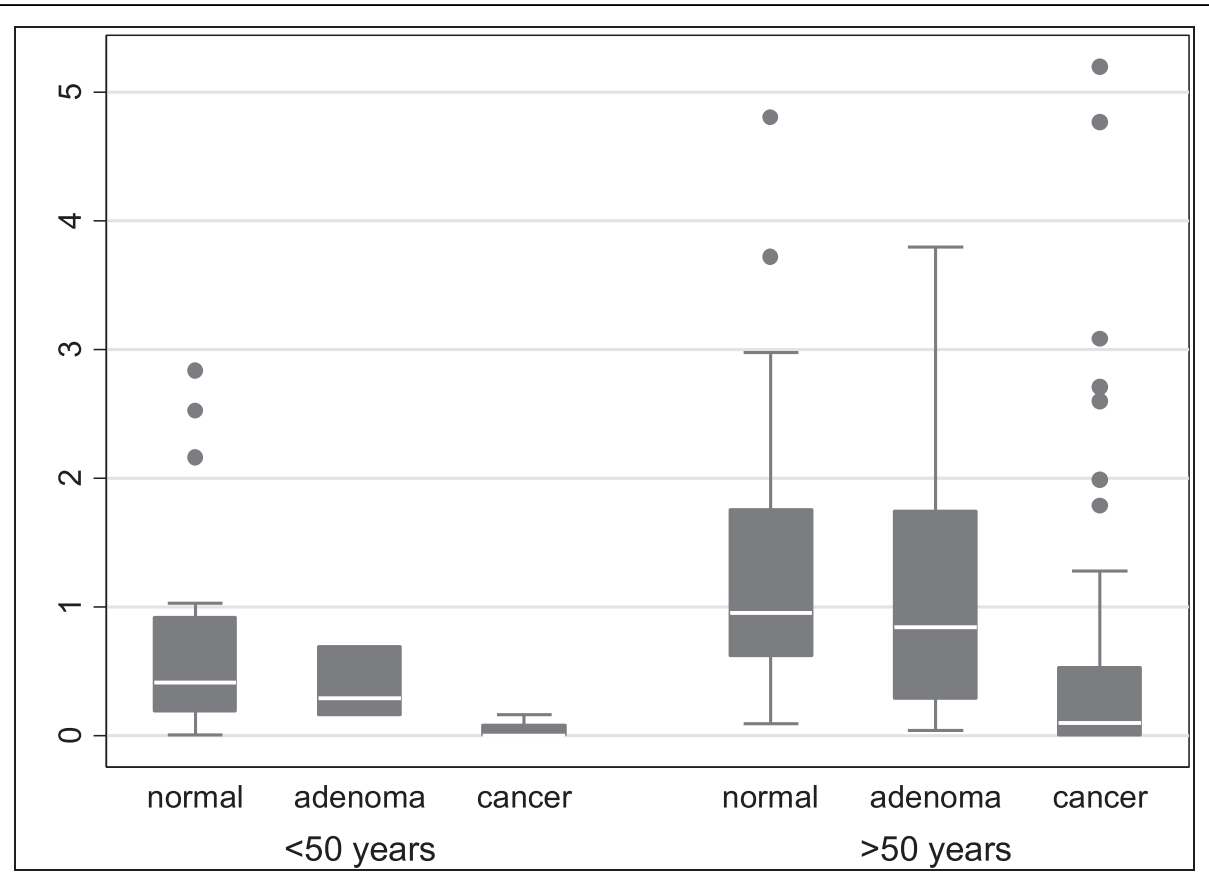

Figure 7 Relationship of THBS4 methylation within the normal mucosa to pathology present elsewhere within the colorectum. THBS4 methylation within the normal mucosa with respect to the pathology present elsewhere within the colorectum was stratified by age $(<50$ years, $p=0.018$ and $>50$ years, $p=0.0001$ ). There was a significant trend evident in both groups $p=0.007$ and $p<0.001$ for young colorectum (<50 yrs) and aged colorectum (> $50 \mathrm{yrs}$ ) respectively, indicating a significant inverse relationship between adenomatous pathology and THBS4 methylation. The lowest levels of THBS4 promoter methylation were associated with the most advanced pathology in both young and aged colorectal mucosa. 
regions of the individual tumours have higher expression. A subset of tumours did express THBS4 and evidently secrete the protein, observed as more intense staining of vesicular structures towards the luminal surface. Indeed, THBS4 can been readily collected from culture supernatants of cells expressing THBS4 constructs [26], or display prominent ER and Golgi labelling [3], indicating that it is a secreted protein. While the secretion of the THBS4 protein may account for the low levels of staining observed, qPCR also demonstrated that gene expression is generally quite low. Thrombospondin4 expression by qPCR is significantly higher in normal tissues than in matched tumour samples, supporting the notion that loss of THBS4 provides a selective growth advantage to cancer cells.

The forced over-expression of THBS4 in cancer cell lines significantly suppresses their colony forming abilities and growth, regardless of their baseline THBS4 expression levels. This was demonstrated in vitro for all $8 \mathrm{CRC}$ cell lines tested, each with varying basal levels of THBS4 expression. A consistent and significant growth suppression of $50-60 \%$ was observed following the forced over-expression of THBS4. This demonstrates that a large amount of THBS4 protein correlates with reduced cell growth, and strengthens the evidence supporting its role as a tumour suppressor gene. Further experiments are now required to investigate the mechanism of this reduced growth, which may be a direct result of THBS4-mediated tumour suppression or toxicity due to higher than physiologically normal levels of THBS4 protein.

The Laird methylation marker panel used to determine CIMP status of colorectal cancers consists of the CACNA1G, IGF2, NEUROG1, RUNX3 and SOCS1 genes [19]. THBS4 methylation in the tumours, as quantitatively measured by MethyLight, significantly increased with the increasing CIMP status of tumours. The greater the number of CIMP markers that were positive for methylation (PMR $\geq 10$ ), the higher the level of THBS4 methylation, although the same proportion of CIMP-L and CIMP-H tumours demonstrated high THBS4 methylation. This suggests that THBS4 methylation may have a role in progression of CIMP positive CRC, regardless of level of CIMP.

We postulated that methylation of the THBS4 promoter region would cause reduction in gene expression level. However, although normal mucosa has significantly higher expression than tumour tissue and significantly lower methylation than tumour tissue, there was no statistically significantly correlation between high promoter methylation and reduced gene expression. Reduced THBS4 expression levels did not correlate with a THBS4 PMR of 10 or greater in either cancers or cell lines. Nevertheless, in another study, methyltransferase inhibition by 5 -azadeoxycytidine in SW48 cells resulted in reactivation of silenced THBS4 [6]. Thus, it appears that other factors may control expression of this gene, and that the moderate levels of THBS4 methylation observed in our study are not enough to significantly inhibit gene expression. It is also possible that other CpG sites within the THBS4 gene promoter that were not examined in this study are important for regulating gene expression

The genes methylated in CRC may be characterized as "type A" (Age-related) genes and "type C" (Cancer-specific) genes [27]. Generally, "type A" genes are methylated in both normal and tumour tissue and their degree of methylation is proportional to the age of the normal tissue $[14,27,28]$. The methylation of "type C" genes, however, is more specific for neoplastic tissue [14,27-30]. From this study, THBS4 behaved as a "type A" gene. THBS4 exhibited methylation in both normal and tumour tissue and displayed a positive correlation with age. Furthermore, as demonstrated in a recent study, [23] THBS4 behaved as a "type A" marker with respect to its association with neoplasia found elsewhere within the field. Methylation of the THBS4 promoter was highest in normal mucosa in patients with normal colonoscopies, and declined progressively as more advanced pathology was evident (Figure 7).

\section{Conclusions}

THBS4 may act as a tumour suppressor gene, evidenced by the dramatic repression of colony formation upon forced over-expression in CRC cell lines and its generally lower expression in cancers compared to normal mucosa. THBS4 methylation is higher in cancers that also exhibit the CIMP phenotype, which is demonstrated by the strong correlation with the Laird panel of methylation markers. However, it appears that methylation has not reached a critical level where it alters THBS4 gene expression, or that THBS4 expression is regulated by factors in addition to promoter hypermethylation. THBS4 promoter methylation occurs in a manner representative of a Type A gene, which is methylated in normal aged colon as part of the aging process. Additional studies are required to identify other genes that are involved in the regulation of THSB4.

\section{List of Abbreviations}

THBS4: Thrombospondin-4; CIMP: CpG Island Methylator Phenotype; $\mathrm{CRC}$ : colorectal cancer

\section{Acknowledgements}

This study was funded by the National Health and Medical Research Council of Australia (grant 442965) and Pathology Queensland, Clinical and

Statewide Services, Queensland Health, Australia. 


\section{Author details}

${ }^{1}$ Conjoint Gastroenterology Laboratory, Royal Brisbane and Women's Hospital Research Foundation, Clinical Research Centre and Queensland Institute of Medical Research, Brisbane, Australia. ${ }^{2}$ Immunovirology Laboratory, Queensland Institute of Medical Research, Brisbane, Australia. ${ }^{3}$ Drug Discovery Group, Queensland Institute of Medical Research, Brisbane, Australia. ${ }^{4}$ Royal Brisbane and Women's Hospital, Brisbane, Australia. ${ }^{5}$ Pathology Queensland, Clinical and Statewide Services, Queensland Health, Brisbane, Australia.

\section{Authors' contributions}

SG contributed to the acquisition of data (expression, methylation and cell line studies), analysis and interpretation of data and drafting of the manuscript.

JC contributed to the acquisition of data (expression and methylation studies), analysis and interpretation of data. KI contributed to the acquisition of data (expression studies), analysis and interpretation of data and drafting of the manuscript. SC contributed to the acquisition of data (expression studies), analysis and interpretation of data. IR contributed to the acquisition of data (CIMP classification) and analysis and interpretation of data. RB contributed to the acquisition of data (methylation in normal mucosa) and analysis and interpretation of data. KS contributed to the acquisition of data (cell line studies), study concept and design. GB contributed to the acquisition of data (cell line studies), study concept and design. DW contributed to the acquisition of data (methylation in normal mucosa), analysis and interpretation of data, statistical analysis and drafting and critical revision of the manuscript. BL contributed to the study concept and design and critical revision of the manuscript. WW contributed to the study concept and design, acquisition of data (expression and methylation studies, immunohistochemistry and cell line studies), drafting and critical revision of the manuscript. All authors have reviewed and approved the final manuscript.

\section{Competing interests}

The authors declare that they have no competing interests.

Received: 16 January 2010 Accepted: 16 September 2010 Published: 16 September 2010

\section{References}

1. Stenina OI, Desai SY, Krukovets I, Kight K, Janigro D, Topol EJ, Plow EF: Thrombospondin-4 and its variants: expression and differential effects on endothelial cells. Circulation 2003, 108(12):1514-1519.

2. Adams JC, Lawler J: The thrombospondins. Int J Biochem Cell Biol 2004, 36(6):961-968.

3. Arber S, Caroni P: Thrombospondin-4, an extracellular matrix protein expressed in the developing and adult nervous system promotes neurite outgrowth. J Cell Biol 1995, 131(4):1083-1094.

4. Carlson CB, Lawler J, Mosher DF: Structures of thrombospondins. Cell Mol Life Sci 2008, 65(5):672-686.

5. Si Z, Palkama A, Gebhardt BM, Velasquez D, Galeano MJ, Beuerman RW: Distribution of thrombospondin-4 in the bovine eye. Curr Eye Res 2003, 27(3):165-173.

6. van Doorn R, Zoutman WH, Dijkman R, de Menezes RX, Commandeur S, Mulder AA, van der Velden PA, Vermeer MH, Willemze R, Yan PS, Huang TH, Tensen CP: Epigenetic profiling of cutaneous T-cell lymphoma: promoter hypermethylation of multiple tumor suppressor genes including BCL7a, PTPRG, and p73. J Clin Oncol 2005, 23(17):3886-3896.

7. Ahuja N, Mohan AL, Li Q, Stolker JM, Herman JG, Hamilton SR, Baylin SB, Issa JP: Association between CpG island methylation and microsatellite instability in colorectal cancer. Cancer Res 1997, 57(16):3370-3374.

8. Korkola JE, DeVries S, Fridlyand J, Hwang ES, Estep AL, Chen YY, Chew KL, Dairkee SH, Jensen RM, Waldman FM: Differentiation of lobular versus ductal breast carcinomas by expression microarray analysis. Cancer Res 2003, 63(21):7167-7175.

9. Kondo Y, Shen L, Yan PS, Huang TH, Issa JP: Chromatin immunoprecipitation microarrays for identification of genes silenced by histone H3 lysine 9 methylation. Proc Natl Acad Sci USA 2004, 101(19):7398-7403.

10. Issa JP: DNA methylation as a therapeutic target in cancer. Clin Cancer Res 2007, 13(6):1634-1637.
11. DNA Methylation in Cancer - Frequently Asked Questions. [http://www. mdanderson.org/departments/methylation/dlndex.cfm?pn=F68B486D-E00C4398-9A7D2FE1F78EC9B9].

12. Toyota M, Ahuja N, Ohe-Toyota M, Herman JG, Baylin SB, Issa JP: CpG island methylator phenotype in colorectal cancer. Proc Natl Acad Sci USA 1999, 96(15):8681-8686.

13. O'Brien MJ, Yang S, Mack C, Xu H, Huang CS, Mulcahy E, Amorosino M, Farraye FA: Comparison of microsatellite instability, CpG island methylation phenotype, BRAF and KRAS status in serrated polyps and traditional adenomas indicates separate pathways to distinct colorectal carcinoma end points. Am J Surg Pathol 2006, 30(12):1491-1501.

14. Ahuja N, Li Q, Mohan AL, Baylin SB, Issa JP: Aging and DNA methylation in colorectal mucosa and cancer. Cancer Res 1998, 58(23):5489-5494.

15. Young J, Jass JR: The case for a genetic predisposition to serrated neoplasia in the colorectum: hypothesis and review of the literature. Cancer Epidemiol Biomarkers Prev 2006, 15(10):1778-1784.

16. Kinzler KW, Vogelstein B: Lessons from hereditary colorectal cancer. Cell 1996, 87(2):159-170.

17. Jass JR, Young J, Leggett BA: Hyperplastic polyps and DNA microsatellite unstable cancers of the colorectum. Histopathology 2000, 37(4):295-301.

18. Kambara T, Simms LA, Whitehall VL, Spring KJ, Wynter CV, Walsh MD, Barker MA, Arnold S, McGivern A, Matsubara N, Tanaka N, Higuchi T, Young J, Jass JR, Leggett BA: BRAF mutation is associated with DNA methylation in serrated polyps and cancers of the colorectum. Gut 2004, 53(8):1137-1144

19. Weisenberger DJ, Siegmund KD, Campan M, Young J, Long TI, Faasse MA, Kang GH, Widschwendter M, Weener D, Buchanan D, Koh H, Simms L, Barker M, Leggett B, Levine J, Kim M, French AJ, Thibodeau SN, Jass J, Haile R, Laird PW: CpG island methylator phenotype underlies sporadic microsatellite instability and is tightly associated with BRAF mutation in colorectal cancer. Nat Genet 2006, 38(7):787-793.

20. Miller SA, Dykes DD, Polesky HF: A simple salting out procedure for extracting DNA from human nucleated cells. Nucleic Acids Res 1988, 16(3):1215.

21. Pfaffl MW: A new mathematical model for relative quantification in realtime RT-PCR. Nucleic Acids Res 2001, 29(9):e45.

22. Eads CA, Danenberg KD, Kawakami K, Saltz LB, Blake C, Shibata D, Danenberg PV, Laird PW: MethyLight: a high-throughput assay to measure DNA methylation. Nucleic Acids Res 2000, 28(8):E32.

23. Worthley DL, Whitehall VL, Buttenshaw RL, Irahara N, Greco SA, Ramsnes I, Mallitt KA, Le Leu RK, Winter J, Hu Y, Ogino S, Young GP, and Leggett BA: DNA methylation within the normal colorectal mucosa is associated with pathway-specific predisposition to cancer. Oncogene, in press

24. Park BH, Vogelstein B: Tumor-Suppressor Genes. Cancer Medicine 6 Hamiton, Ontario: BC Decker Inc 2003, 87-102.

25. Grady WM, Carethers JM: Genomic and epigenetic instability in colorectal cancer pathogenesis. Gastroenterology 2008, 135(4):1079-1099.

26. Lawler J, McHenry K, Duquette M, Derick L: Characterization of human thrombospondin-4. J Biol Chem 1995, 270(6):2809-2814.

27. Toyota M, Issa JP: CpG island methylator phenotypes in aging and cancer. Seminars in cancer biology 1999, 9(5):349-357.

28. Ahuja N, Issa JP: Aging, methylation and cancer. Histol Histopathol 2000, 15(3):835-842.

29. Shen L, Kondo Y, Rosner GL, Xiao L, Hernandez NS, Vilaythong J, Houlihan PS, Krouse RS, Prasad AR, Einspahr JG, Buckmeier J, Alberts DS, Hamilton SR, Issa JP: MGMT Promoter Methylation and Field Defect in Sporadic Colorectal Cancer. J Natl Cancer Inst 2005, 97(18):1330-1338.

30. Issa JP, Ahuja N, Toyota M, Bronner MP, Brentnall TA: Accelerated agerelated CpG island methylation in ulcerative colitis. Cancer Res 2001, 61(9):3573-3577.

\section{Pre-publication history}

The pre-publication history for this paper can be accessed here: http://www.biomedcentral.com/1471-2407/10/494/prepub

\section{doi:10.1186/1471-2407-10-494}

Cite this article as: Greco et al:: Thrombospondin-4 is a putative tumoursuppressor gene in colorectal cancer that exhibits age-related methylation. BMC Cancer 2010 10:494. 